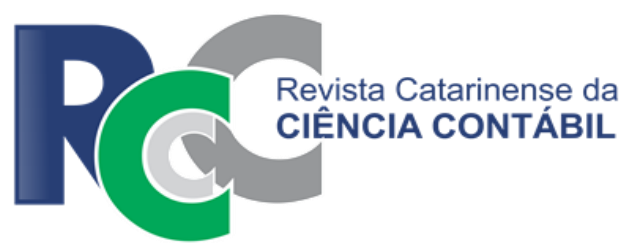
maio/ago. 2017

\title{
CAPITAL HUMANO E SUA RELAÇÃO COM O DESEMPENHO ORGANIZACIONAL EM EMPRESAS PRESTADORAS DE SERVIÇOS CONTÁBEIS
}

\author{
HUMAN CAPITAL AND ITS RELATIONSHIP WITH THE ORGANIZATIONAL \\ PERFORMANCE IN ACCOUNTING SERVICES PROVIDERS
}

\author{
SUZETE ANTONIETA LIZOTE \\ Doutora em Administração e Turismo. Professora do Programa Pós- \\ Graduação em Administração e Graduação em Ciências Contábeis na \\ Universidade do Vale do Itajaí. Endereço: Av. Marcos Konder, 1.100 / \\ Apto. 801 / 88301-302 | Itajaí/SC / Brasil. \\ E-mail: lizote@univali.br
}

\section{CLÁUDIA SILVA RIBEIRO ALVES}

Mestranda em Administração. Professora de Graduação do Curso de Administração na Universidade do Vale do Itajaí. Endereço: Rua Camboriú, 689, apto 04 | Fazenda | 88330-002 | Itajaí/SC | Brasil.

E-mail: dinha.csra@gmail.com

\section{MIGUEL ANGEL VERDINELLI}

Doutor em Ciências. Professor do Programa Pós-Graduação em Administração na Universidade do Vale do Itajaí. Endereço: Rua Dr. Francisco Rangel, 05 | Fazenda | 88302-662 | Itajaí/SC | Brasil.

E-mail: nupad@univali.br

\section{JOSÉ CARLOS TERRES}

Mestre em Contabilidade. Professor e Coordenador do Curso de Graduação em Ciências Contábeis na Universidade do Vale do Itajaí. Endereço: Av. Marcos Konder, 1.100 | Apto. 801 | 88301-302 | Itajaí/SC / Brasil.

E-mail: jcterres@terra.com.br

\section{RESUMO}

$\mathrm{Na}$ atualidade o valor das empresas não pode ser estimado apenas pelos seus ativos tangíveis. A geração e aquisição de conhecimento são fundamentais para a obtenção de vantagens competitivas, pelo que cresce a importância do capital intelectual da organização. Nesse contexto a pesquisa objetiva analisar se o capital humano $(\mathrm{CH})$ que as empresas prestadoras de serviços contábeis dispõem se relaciona positivamente com seu desempenho organizacional (DO). Metodologicamente, a pesquisa se caracteriza como quantitativa e de natureza aplicada, com os dados empíricos obtidos com questionário numa amostra intencional. Levantaram-se dados de ambos os constructos e dos respondentes e suas empresas. Os métodos estatísticos foram o teste $t$ e a análise de variância para as comparações de médias. Empregou-se também a análise fatorial exploratória e confirmatória e a modelagem em equações estruturais para avaliar a relação conjecturada. Os resultados obtidos mostraram que os respondentes têm percepções estatisticamente iguais para ambos os constructos ao considerar o gênero e o tipo de gestão que a firma possui, mas houve diferenças para a percepção do desempenho segundo seu tamanho. Empresas maiores 
declaram ter maior desempenho. A hipótese que $\mathrm{o} C H$ se relaciona positiva e significativamente com o DO foi confirmada.

Palavras-chave: Capital Humano. Desempenho. Contabilidade.

\begin{abstract}
Currently the value of companies cannot only be estimated by their tangible assets. The generation and acquisition of knowledge are key for obtaining competitive advantages, reason for which the importance of the intellectual capital of the organization grows. In this context the research aims to analyze whether the human capital $(\mathrm{CH})$ held by companies providing accounting services are positively related to their organizational performance (DO). Methodologically, the research is characterized as quantitative and of an applied nature, with the empirical data obtained from a questionnaire in intentional sample. Data were collected from both constructs and respondents and their companies. Statistical methods were $t$ test and the analysis of variance for averages comparisons. We have also used exploratory and confirmatory factor analysis and modeling in structural equations to evaluate the conjectured relationship. The results have showed that the respondents have statistically equal perceptions for both constructs when considering gender and the type of management the firm has, but there were differences for the perception of performance according to the size. Larger companies report higher performance. The hypothesis that $\mathrm{CH}$ is positively and significantly related to $O D$ is, therefore, confirmed.
\end{abstract}

Keywords: Human Capital. Performance. Accounting.

\title{
1 INTRODUÇÃO
}

$\mathrm{Na}$ atual conjuntura econômica, especialmente com a globalização dos mercados, os ativos que se estão valorizando mais são os intangíveis. $E$, dentre eles, o capital intelectual tornou-se um dos mais relevantes nos estudos do desempenho organizacional por ser fonte de vantagens competitivas (Curado \& Bontis, 2007).

O conceito de capital intelectual está relacionado ao papel que desempenha o conhecimento no crescimento econômico (Huang \& Liu, 2005) e sua natureza multidisciplinar tem gerado diversas perspectivas de análise, bem como dificuldades para sua avaliação (Bontis, Dragonetti, Jacobsen, \& Roos, 1999). A diferença entre o valor do mercado das empresas e seu valor contábil foi um dos primeiros determinantes que gerou interesse no tema.

Em face de tais condições as organizações tiveram necessidade de dispor de ferramentas adequadas para medir o valor dos seus recursos intangíveis (Bontis et al., 1999). Pois, embora os bens tangíveis de uma companhia contribuam no valor final da sua produção ou serviço, a maior parcela deve-se ao "talento dos seus colaboradores, a eficácia dos seus sistemas de gestão, as modalidades do seu relacionamento com os clientes - tudo isto constitui em conjunto o seu capital intelectual" (Stewart, 1998, p. 87).

O conceito de capital intelectual é empregado com frequência como sinônimo de ativo intangível. Contudo, em algumas definições, por exemplo, a da Society of Management Accountants of Canada, não se inclui a competência dos colaboradores, a imagem e reputação da empresa, a sua organização e cultura, a fidelização dos seus clientes, dentre outros componentes (Lopes Costa, 2012), aspectos esses que se relacionam ao conceito multidimensional de capital intelectual. Para o constructo se reconhecem três dimensões: capital humano, capital estrutural e capital relacional (Edvinsson \& Malone, 1997).

O componente de maior valor das dimensões do capital intelectual, segundo Rahman (2012), é o capital humano e as organizações com grande eficiência nesse tipo de capital tendem a mostrar melhor performance financeira.

No caso das empresas prestadoras de serviços contábeis, tanto o capital estrutural quanto o relacional podem ser considerados como relativamente constantes. A organização do trabalho, a relações entre chefes e subordinados, a aprendizagem organizacional como 
exemplos de aspectos estruturais, flutuam pouco através do tempo. Já a tecnologia da informação, que dos componentes estruturais é o mais mutável, em geral relacionado a obrigações normatizadas, acaba afetando a todos os escritórios por igual e assim o efeito se dilui. Do mesmo modo a carteira de clientes, sua satisfação, os índices de queixas, como alguns dos itens próprios das relações com os usuários dos serviços, também se mantêm quase estáveis.

No entanto, um fato comum é o alto turnover que se verifica nessas empresas. A rotatividade dos colaboradores que constituíram os sujeitos desta pesquisa se reflete na relação que há entre o tempo na profissão comparado com o tempo na empresa em que atualmente trabalham. Portanto, justifica-se estudar a dimensão que mensura o capital humano, concebido como o conhecimento, competências e capacidades dos indivíduos (Coleman, 1988).

Levando em conta essa asseveração pode-se afirmar que toda organização possui seu capital humano, mas o êxito empresarial passa também por outros condicionantes. Sem dúvidas a capacidade de absorção de conhecimento, ou seja, a "capacidade de uma empresa em reconhecer o valor da informação nova, externa, assimilá-la e aplicá-la para fins comerciais é fundamental para suas capacidades inovadoras" (Cohen \& Levinthal, 1990, p. 128), constitui um dos pilares do sucesso.

Com base no contexto acima descrito, este trabalho tem como objetivo analisar se o capital humano $(\mathrm{CH})$ que as empresas prestadoras de serviços contábeis dispõem se relaciona positivamente com seu desempenho organizacional (DO).

Com o presente estudo buscou-se obter novas evidências empíricas a respeito das relações entre os construtos analisados. Sua consecução, além de original para as organizações foco da pesquisa, possui relevância prática para a melhoria do funcionamento dos escritórios. Por outro lado, as proposições de articulações teóricas, ainda pouco trabalhadas em estudos empíricos da realidade brasileira, justificam o interesse acadêmico.

A seguir desta introdução, é apresentado o marco teórico definido. Na seção seguinte se detalha o material e métodos utilizados, seguidos da descrição e análise dos dados. Por último, são feitas as considerações finais e disponibilizado o referencial bibliográfico citado no texto.

\section{REFERENCIAL TEÓRICO}

Nesta seção apresenta-se o marco teórico definido como necessário à compreensão da abordagem adotada. Depois do tratamento do constructo capital humano formula-se a hipótese de sua relação com o desempenho organizacional nas empresas prestadoras de serviços contábeis.

\subsection{Capital intelectual}

O valor de uma organização vem sendo influenciado diretamente pelo conhecimento e sua aplicação, assegurando dessa forma benefícios. Genericamente têm sido denominados como ativos intangíveis e também como capital intelectual. Wernke, Lembeck e Bornia (2003, p. 24) mencionam que "apesar da dificuldade de compreensão e percepção do capital intelectual em face da sua subjetividade, resta evidente que ele é representativo e importante atualmente, repercutindo no valor de mercado das empresas". Cassol, Zapalai e Cintra (2017, p. 13), por sua vez, destacam que "As organizações começaram a buscar possibilidades de registrar e gerenciar seus ativos intangíveis com o objetivo de desenvolver diferenciais competitivos em relação aos seus concorrentes, intensificando o valor da gestão do conhecimento".

O conceito desse capital se relaciona ao papel que desempenha o conhecimento no crescimento econômico (Huang \& Liu, 2005). Assim, tem-se assinalado que o capital intelectual é um ativo vital para o sucesso organizacional (Bontis, Keow, \& Richardson, 2000; De Castro, Sáez, 2008, Kamukam, Ahiauzu, \& Ntayi, 2010). Stewart (1998, p. 8) argumenta que "o capital intelectual constitui a matéria intelectual - conhecimento, informação, propriedade intelectual, experiência, que pode ser utilizada para gerar riqueza. É a capacidade mental coletiva". 
Depreende-se que o capital intelectual pode ser considerado como a soma de conhecimentos, seja individual ou coletivo, bem como tácito ou explícito (Bontis, 2001), que as empresas utilizam para obter vantagem competitiva. Devido ao fato de resultar dessa soma, os intangíveis que o compõe são condutores do desempenho organizacional (Sainaghi \& Baggio, 2013).

Na visão de Perez e Famá (2006) o capital intelectual é gerado pela inovação, por práticas organizacionais e pelos recursos humanos. Segundo Gracioli, Godoy, Lorenzett e Godoy (2012), ele é responsável por uma porcentagem cada vez maior no desenvolvimento das organizações, criando a maior parte do valor de seus produtos e serviços. Em termos gerais, os autores expressam que todos os recursos intangíveis e suas interconexões são considerados capital intelectual. Brooking (1996), Stewart (1998), Sveiby (1998) e Edvinsson e Malone $(1997,1998)$ entendem que o capital intelectual é formado por um conjunto de componentes, conforme a Figura 1.

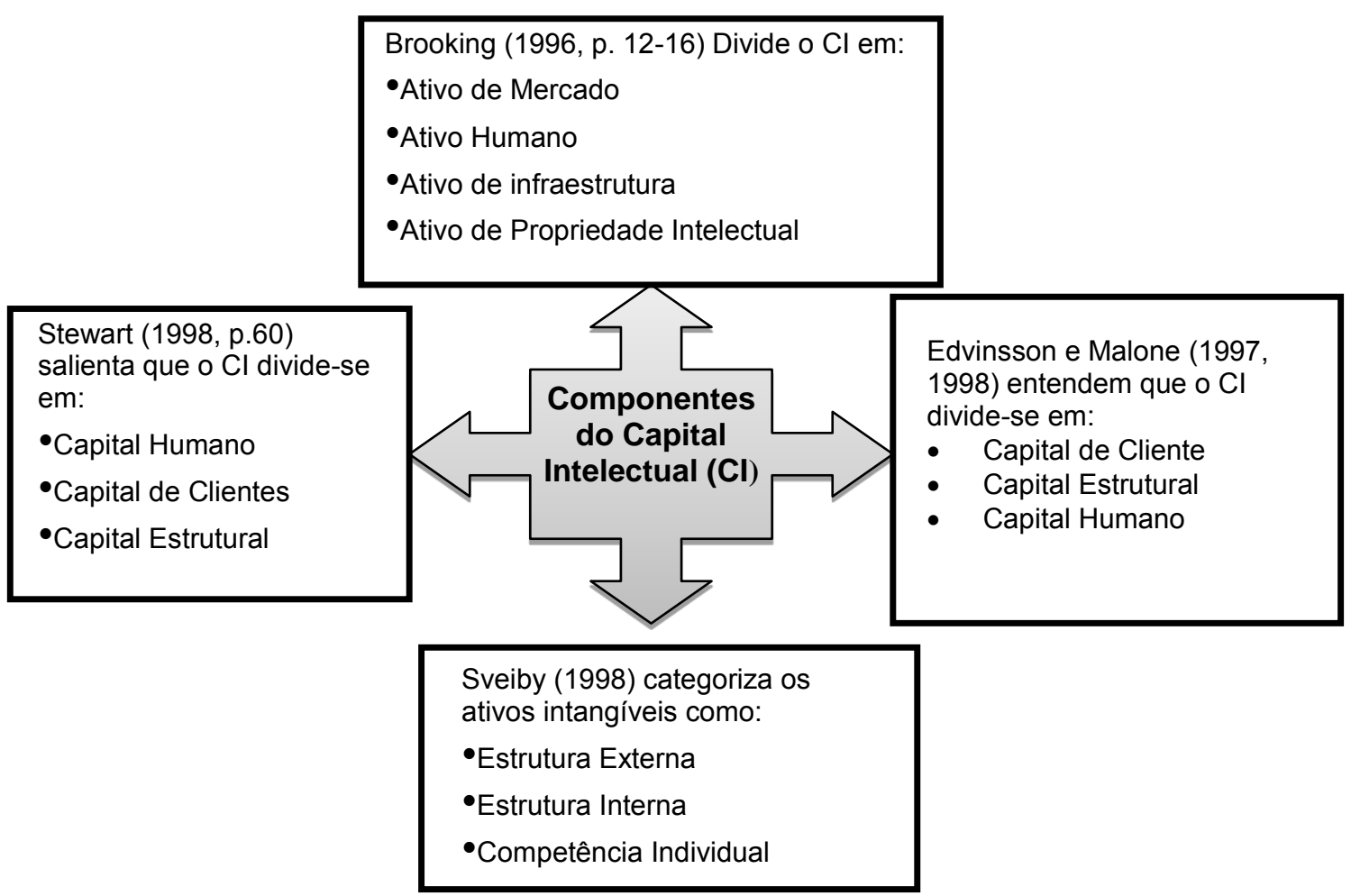

Figura 1. Componentes do Cl segundo Brooking (1996), Stewart (1998), Sveiby (1998) e Edvinsson e Malone $(1997,1998)$

Fonte: (Edvinsson \& Malone, 1997, 1998).

Em relação à Figura 1, esta pesquisa filia-se teoricamente à categorização de capital intelectual proposta por Edvinsson e Malone (1997, 1998), que entendem que o Cl é composto pelo capital humano, estrutural e relacional. Na sequência, abordam-se de maneira mais aprofundada capital humano por ser o componente analisado neste estudo. 


\subsection{Capital Humano}

O valor de uma organização vem sendo influenciado diretamente pelo conhecimento e sua aplicação, assegurando dessa forma benefícios. Genericamente têm sido denominados como ativos intangíveis e também como capital intelectual. $O$ conceito deste capital se relaciona ao papel que desempenha o conhecimento no crescimento econômico (Huang \& Liu, 2005). Assim, tem-se assinalado que o capital intelectual é um ativo vital para o sucesso organizacional (Bontis, Keow, \& Richardson, 2000, De Castro \& Sáez, 2008). A ciência contábil tem como desafio mensurá-lo, com o propósito de evidenciar o retorno que esses ativos intangíveis proporcionam para a empresa (Oliveira \& Beuren, 2003, Sena \& Petri, 2011).

Nesse sentido, argumenta Stewart (1998, p. 8), que "o capital intelectual constitui a matéria intelectual - conhecimento, informação, propriedade intelectual, experiência, que pode ser utilizada para gerar riqueza. É a capacidade mental coletiva". O capital intelectual pode ser considerado então como a soma de conhecimentos, seja individual ou coletivo, bem como tácito ou explícito (Bontis, 2001), que as empresas utilizam para obter vantagem competitiva ao serem convertidos em valor (Silva, Bilich, \& Gomes, 2002).

Na visão de Perez e Famá (2006), o capital intelectual é gerado pela inovação, por práticas organizacionais e pelos recursos humanos. Em termos gerais, os autores expressam que todos os recursos intangíveis e suas interconexões são considerados capital intelectual, o qual é formado, segundo propuseram Edvinsson e Malone (1997), pelos componentes: capital estrutural, relacional e humano. Esse último será o centro de convergência deste estudo.

O capital humano é um ativo intangível que pode ser utilizado para gerar valor para a organização. Assim, argumenta Florin (2005) que o capital humano refere-se tanto à capacidade, habilidade e experiência quanto ao conhecimento formal que as pessoas detêm e que agregam valor à empresa. Ou seja, conforme fora concebido por Coleman (1988), ele compreende o conhecimento, as competências e as capacidades dos indivíduos. Gil e Arnosti (2007) salientam que o capital humano envolve os conhecimentos e a capacidade de aprendizagem dos colaboradores que constituem a organização. Portanto, os investimentos em capital humano, segundo afirmam Unger, Rauch, Frese e Rosenbusch (2009), tornam-se importantes porque possibilitam às empresas melhoria no grau de capacitação de seus colaboradores, aumentando a sua satisfação, dedicação e, consequentemente, o desempenho. Assim, é possível estabelecer a seguinte hipótese:

$\mathrm{H} 1$ : O capital humano se relaciona positivamente com o desempenho organizacional.

\subsection{Desempenho Organizacional}

A avaliação de desempenho serve para controlar uma estratégia definida pela organização, confrontando seu resultado com os objetivos estabelecidos. Nessa ótica, Neely, Gregory e Platts (2005) a veem como um processo de quantificação da eficiência e efetividade das ações empresariais. Para Igarashi, Ensslin, Ensslin e Paladini (2008), é mediante a análise do desempenho que as organizações podem medir a sua capacidade de sobrevivência e continuidade, face às exigências do ambiente interno e externo em que estejam inseridas. $O$ crescimento do interesse pelas medidas de desempenho organizacional deve-se às importantes mudanças tanto no ambiente empresarial quanto nas estratégias adotadas (Mcadam \& Bailie, 2002).

No que tange a sua mensuração, Gunasekaran e Kobu (2007) consideram um grande desafio para os administradores o desenvolvimento de medidas apropriadas para a tomada de decisões que contribuam para o alcance da competitividade. Backes, Silva, Adão e Corso, (2009) argumentam que medir o desempenho é complexo e devem ser levadas em consideração as várias formas metodológicas e abordagens utilizadas na sua avaliação. $O$ desempenho pode ser mensurado por meio de duas perspectivas: primeiramente como conceito subjetivo, o qual está relacionado ao desempenho das organizações segundo a sua própria expectativa ou relativamente à concorrência (Pelham \& Wilson, 1996). A segunda opção é analisá-lo pelo método objetivo, baseado em medidas absolutas de desempenho (Chakravarthy, 1996).

Para dar suporte às decisões estratégicas da organização, sua mensuração, segundo Bortoluzzi, Ensslin e Ensslin (2010), deve considerar alguns elementos importantes, quais 
sejam: levar em consideração as particularidades de cada organização; considerar indicadores financeiros e não financeiros, ou seja, os aspectos tangíveis e intangíveis; ligar os objetivos estratégicos com os objetivos operacionais; e, construir um processo de comunicação que permita a todos os níveis organizacionais identificar de forma clara e holística os objetivos que a organização está idealizando. Neste sentido, para Dani, Santos e Kaveski (2017, p. 35) "[...] os indicadores de desempenho empresarial são elaborados com o propósito de fornecer suporte necessário aos tomadores de decisão e a sobrevivência da organização".

Entretanto, a validade de usar indicadores subjetivos de desempenho tem sido demonstrada como alternativa viável para o caso de inexistência de dados secundários confiáveis (Perin \& Sampaio, 1999) ou a impossibilidade de obtê-los. Conforme comenta Hoque (2005), os motivos pelos quais geralmente são escolhidas as medidas subjetivas ocorrem porque uma parcela significativa de pequenas empresas não possuem aqueles dados ou porque dispõem de poucas informações objetivas, tornando quase que impossível conferilas com precisão. Neste estudo, escolheu-se utilizar medidas subjetivas de desempenho, trabalhando com a percepção dos gestores dos escritórios de contabilidade. Essa decisão se baseia no fato de que esse constructo constitui a variável dependente no modelo e sua mensuração, como um valor em relação aos concorrentes mais próximos, é apropriada para estimar as relações que com ele tem o capital humano $(\mathrm{CH})$.

\section{MATERIAL E MÉTODOS}

Diante dos critérios adotados, a abordagem do problema foi quantitativa. A avaliação quantitativa compreende organizar, sumarizar, caracterizar e interpretar os dados numéricos (Martins \& Theóphilo, 2007). As características deste estudo, do ponto de vista dos seus objetivos, o posiciona como descritivo. Para Vergara (1998), os objetivos da pesquisa descritiva referem-se à obtenção de informações sobre um fenômeno ou sobre determinada população e descrição de suas características. No que tange aos procedimentos técnicos, foi do tipo levantamento, que tem como objetivo contribuir para o conhecimento de uma área particular de interesse por meio da coleta de dados sobre indivíduos ou sobre o seu ambiente.

O material para o estudo foi obtido por meio de uma amostra não aleatória usando um questionário de autopreenchimento. Ele foi aplicado nos meses de março e abril de 2016 pessoalmente e também enviado via correio eletrônico aos gerentes, coordenadores e assistentes de empresas prestadoras de serviços contábeis localizadas nos municípios de Itajaí, Balneário Camboriú e Itapema do Estado de Santa Catarina. O instrumento de coleta de dados esteve composto por 33 asseverações, a serem respondidas com uma escala Likert de concordância de 7 pontos, indo desde discordo totalmente (1) até concordo plenamente (7). Dispuseram-se também 6 questões para obter dados dos respondentes e dos escritórios.

As duas seções principais do questionário compreendiam os constructos: capital humano $(\mathrm{CH})$ e desempenho organizacional (DO). A primeira seção continha 26 asseverações para o $\mathrm{CH}$, mensurado pelos valores (4), atitudes (5), conhecimentos (6), capacidades (6) e pela liderança (5). Na última seção, destinada a mensurar a percepção sobre o DO, dispuseram-se 7 itens a serem avaliados em relação com o concorrente mais próximo. Os indicadores foram: lucro líquido, vendas totais, realização dos objetivos financeiros, metas de emprego e de pessoal, satisfação de clientes, retenção de clientes e desempenho geral.

Todos os dados obtidos foram digitados numa planilha eletrônica Excel $\AA^{\circledR}$, na qual inicialmente fez-se o pré-processamento segundo as indicações de Hair, Black, Babin, Anderson e Tatham (2009). Observou-se que existiam 17 dados faltantes nos 202 questionários recebidos, mas como não foi reconhecida a ocorrência de qualquer padrão foram preenchidos com o valor da mediana do item considerado. A seguir, avaliaram-se os outliers usando a função gráfica Box-Plot do software Statistica ${ }^{\circledR}$, com a que foram reconhecidos 32 . Ao igual que com os dados faltantes verificou-se que não seguiam algum padrão e se optou por mantê-los. Não se registraram erros de digitação. Como resultado dos procedimentos descritos a base de dados inicial ficou composta por 202 respondentes e 39 variáveis.

Os métodos estatísticos usados para comparar médias foram o teste $t$ e análise de variância. A Anova realizada foi univariada e, portanto, muito robusta frente às violações de 
normalidade e homocedasticidade (Harris, 1975). Os métodos multivariados empregados para avaliar as relações entre os constructos foram análise fatorial exploratória (AFE), análise fatorial confirmatória (AFC) e modelagem de equações estruturais (MEE). Os softwares usados foram o Statistica ${ }$, SPSS $®$ e o $A M O S \circledR$.

Antes de realizar as análises fatoriais foi calculado o coeficiente alfa de Cronbach para cada constructo considerado e a correlação do item com o total, conforme o procedimento sugerido por Churchill (1979). Calculou-se ainda a correlação média interitens e realizaram-se o teste de Kaiser, Olkin e Meyer (KMO) e o de Bartlett para confirmar a factibilidade de empregar a análise fatorial. Na AFE usou-se a extração por componentes principais, que não requer multinormalidade. Os fatores foram extraídos segundo o critério de Kaiser para matrizes de correlação.

Outras restrições impostas foram que as cargas fatoriais fossem maiores ou iguais do que 0,70 em módulo e a comunalidade maior ou igual que 0,5 . A variância extraída pelo fator no caso de unidimensionalidade devia ser maior ou igual que 50\%. Depois desses procedimentos a base ficou com 35 variáveis. Incluem-se nelas as 6 variáveis referidas às informações individuais e empresariais junto com as 23 medindo o capital humano e 6 o desempenho. Com a finalidade de avaliar a normalidade da distribuição dessas variáveis selecionadas e ao levar em conta que os dados provinham de escalas Likert, efetuaram-se os cálculos da assimetria e curtose (Hair et al., 2009). Finney e Distefano (2006) afirmam que dados com coeficientes de até 2 de assimetria e até 7 de curtose, em módulo, podem ser considerados quase normais.

Confirmado que cada fator extraído esteve com mais de três itens foi desenvolvida a análise fatorial confirmatória (AFC) empregando-se o programa AMOS $\otimes$. Colocou-se como restrição que entre o indicador e a dimensão ou constructo avaliado o coeficiente padronizado devia ter um valor mínimo de 0,50. A AFC corrige possíveis deficiências do modelo exploratório e conduz a uma maior certeza das hipóteses que devem ser contrastadas por meio de modelos que expliquem os inter-relacionamentos existentes na estrutura de um questionário. Neste estudo utilizou-se a AFC para validar o modelo de mensuração de modo individual para cada dimensão do constructo e, a seguir, para o constructo considerando todas as suas dimensões. Finalmente se validou o modelo geral de mensuração entre o capital humano e o desempenho.

A análise da relação conjecturada foi realizada por meio da modelagem de equações estruturais, também com o software $A M O S \circledR$. A função principal da MEE e a especificação e estimação de modelos de relações lineares entre variáveis. Essa técnica oferece a possibilidade de investigar quão bem as variáveis preditoras explicam a variável dependente e também é possível identificar qual das variáveis preditoras é a mais importante (Kline, 2011).

\section{RESULTADOS}

Com os dados da amostra obtida, que compreendeu 202 questionários válidos e 35 variáveis, previamente a efetuar as análises fatoriais se realizaram os procedimentos descritos na seção anterior. Assim, seguindo o sugerido por Churchill (1979), para confirmar a adequação dos dados, avaliou-se a confiabilidade por meio do alfa de Cronbach e as correlações do item com o total, além do valor médio da correlação inter itens. Os resultados obtidos se apresentam na Tabela 1, em que consta ainda o valor do teste de Kaiser, Olkin e Meyer (KMO), que também serve para confirmar a factibilidade de efetuar uma análise fatorial. Espera-se para o KMO valores acima de 0,7 . Os resultados do teste de Bartlett não se apresentam na Tabela uma vez que todos eles foram significativos $(p<0,001)$. 
Tabela 1

Indicadores de factibilidade de efetuar uma análise fatorial com os constructos considerados

\begin{tabular}{|c|c|c|c|c|c|c|}
\hline \multirow{2}{*}{ Constructo } & \multirow{2}{*}{ Dimensão } & \multirow{2}{*}{$\begin{array}{l}\text { Número } \\
\text { de itens }\end{array}$} & \multirow{2}{*}{$\begin{array}{l}\alpha \quad \text { de } \\
\text { Cronbach }\end{array}$} & \multicolumn{2}{|c|}{ Correlação } & \multirow{2}{*}{ K-M-O } \\
\hline & & & & Item-total & Inter-itens & \\
\hline \multirow{5}{*}{$\mathrm{CH}$} & Valores & 4 & 0,8428 & $>0,6$ & 0,576 & 0,781 \\
\hline & Atitudes & 5 & 0,9196 & $>0,7$ & 0,703 & 0,884 \\
\hline & Conhecimentos & $5 *$ & 0,8622 & $>0,4$ & 0,527 & 0,819 \\
\hline & Capacidades & 5 * & 0,8181 & $>0,45$ & 0,484 & 0,758 \\
\hline & Liderança & 5 & 0,9049 & $>0,65$ & 0,682 & 0,787 \\
\hline DO & Desempenho & $6^{*}$ & 0,9420 & $>0,55$ & 0,729 & 0,876 \\
\hline
\end{tabular}

Nota. Fonte: Dados da pesquisa (2016).

* significa que o número de itens foi ajustado para obter valores satisfatórios.

As análises fatoriais exploratórias efetuadas possibilitaram fazer outros ajustamentos na base de dados resultando no número final de itens por dimensão e de cada constructo considerado (Tabela 1). Calculada a assimetria e a curtose verificou-se que nenhum dos valores ultrapassa os limites sugeridos por Finney e Distefano (2006), como se exibe na Tabela 2. Isso indica que a distribuição pode ser considerada quase normal.

Definidas as variáveis utilizadas na análise de dados fez-se uma comparação de médias para cada constructo. Para tanto, a variáveis dependentes foram as somatórias das pontuações atribuídas aos indicadores selecionados de cada constructo. $\mathrm{Na}$ comparação entre respondentes usou-se como variável categórica o gênero e para contrastar as empresas empregou-se o tipo de administração que elas apresentam: familiar ou profissional. No primeiro teste $t$ não se verificaram diferenças ligadas ao gênero do sujeito da pesquisa para nenhum dos constructos. Para o tipo de gestão houve diferença nas médias de três dimensões do capital humano, com valores maiores nas empresas de gestão familiar. Essas diferenças se verificaram para as atitudes, capacidades e liderança, porém para o somatório total das cinco dimensões houve igualdade.

Ao considerar o tamanho do escritório, que se categorizou em pequeno (até 49), médio (de 50 a 99) e grande (com 100 ou mais funcionários), o contraste das médias fez-se empregando a análise de variância. Nas comparações simultâneas houve diferenças para o desempenho e para o capital humano, mensurados ambos os constructos pela soma total dos indicadores selecionados (Tabela 2).

Tabela 2

\section{Cálculo das medidas descritivas dos itens selecionados nas análises fatoriais} exploratórias

\begin{tabular}{|c|c|c|c|c|c|c|c|c|c|c|c|c|}
\hline Dimensão & Item & Méd & d.p. & Ass. & Curtose & & Dimensão & Item & Méd & d.p. & Ass. & Curtose \\
\hline \multirow{6}{*}{ 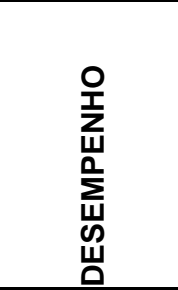 } & D01 & 5,38 & 201 & 0,08 & $-1,317$ & \multirow{7}{*}{ 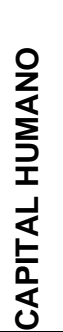 } & \multirow{5}{*}{ 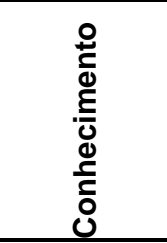 } & CN1 & 5,34 & 1,076 & $-0,41$ & 0,025 \\
\hline & DO2 &, 57 & 085 & $-0,06$ & 9 & & & CN3 & 5,22 & ,178 & 0,03 & $-0,751$ \\
\hline & DO4 & 5,73 & 1,131 & $-0,31$ & $-1,305$ & & & CN4 & 5,52 & 1,217 & $-0,24$ & $-0,849$ \\
\hline & DO5 & 5,80 & 1,157 & $-0,46$ & $-1,253$ & & & CN5 & 5,77 & 1,023 & $-0,51$ & $-0,540$ \\
\hline & D06 & 5,80 & 1,215 & $-0,46$ & $-1,384$ & & & CN6 & 5,30 & 1,087 & $-0,19$ & $-0,777$ \\
\hline & DO7 & 5,77 & 150 & $-0,40$ & $-1,286$ & & \multirow{2}{*}{$\stackrel{+}{+}$} & AT1 & 5,83 & 1,293 & $-0,91$ & $-0,130$ \\
\hline Dimensão & Item & Méd & d.p. & Ass. & Curtose & & & AT2 & 5,77 & 1,144 & $-0,70$ & $-0,070$ \\
\hline
\end{tabular}


Tabela 2 (continuação)

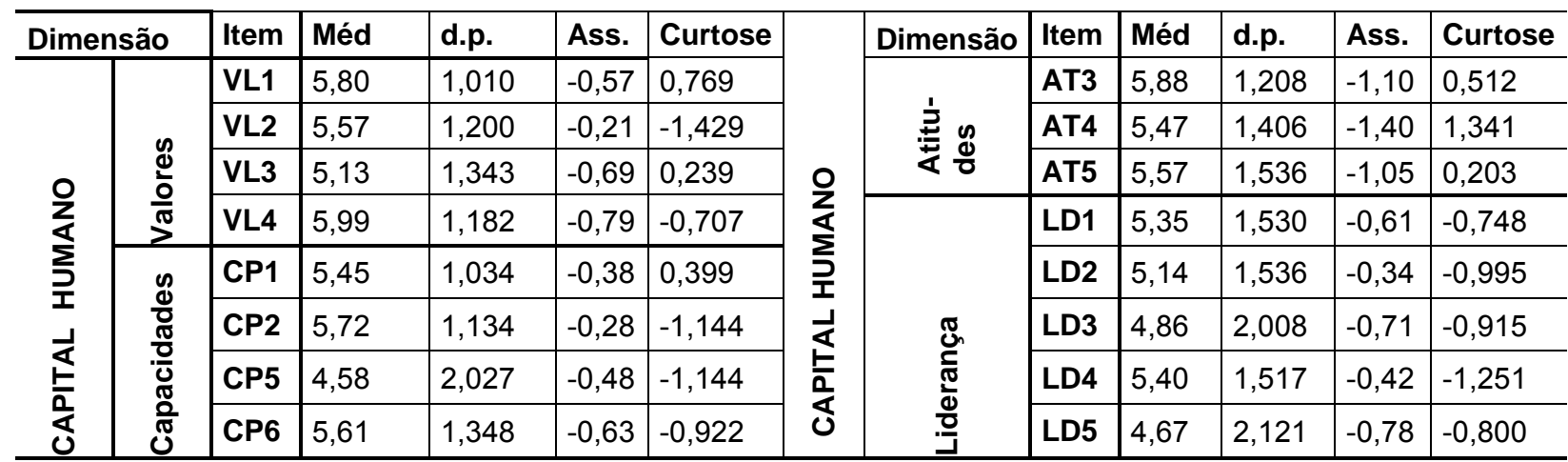

Nota. Fonte: Dados da pesquisa (2016).

$\mathrm{Na}$ Anova para o capital humano a comparação simultânea mostrou que existem diferenças entre as médias, conforme se exibe na Figura 2 e confirma o resultado do teste [F(2, $199)=3,4968, p=0,03217]$. Entretanto, ao efetuar o contraste das comparações pareadas com o teste de Tukey para número desigual de observações, nota-se que as empresas grandes têm pontuações médias maiores do que as pequenas, mas sua significância só se verifica aos $10 \%(p=0,067)$. Por sua vez as empresas médias não se diferenciam nem das grandes nem das pequenas.

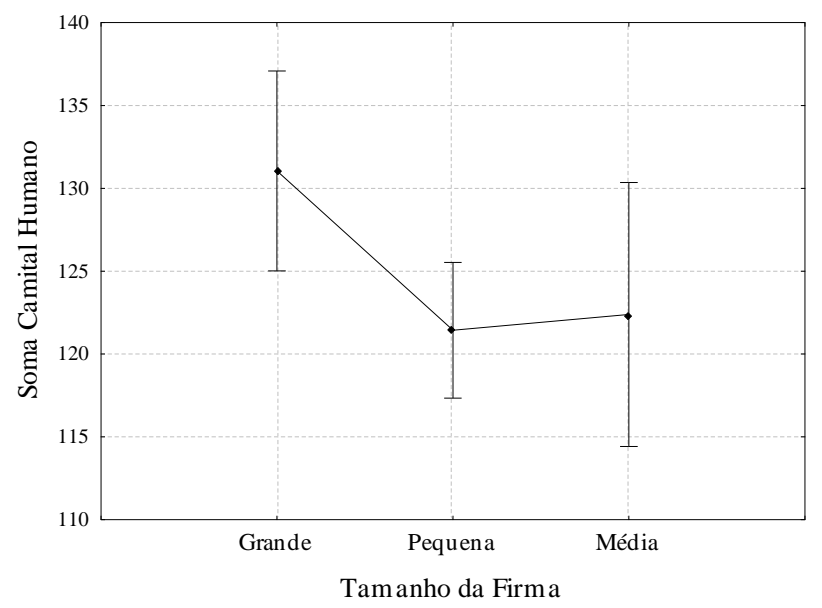

Figura 2. Comparação simultânea na Anova da somatória da pontuação dos itens selecionados do capital humano segundo o tamanho do escritório de prestação de serviços contábeis

Fonte: Dados da pesquisa (2016).

Por sua vez, na Anova do desempenho com os mesmos preditores também se confirma que há diferenças na comparação simultânea $[F(2,199)=4,6322, p=0,01081]$. De igual modo, são os escritórios de maior tamanho os que declaram ter melhor desempenho (Figura 3). 


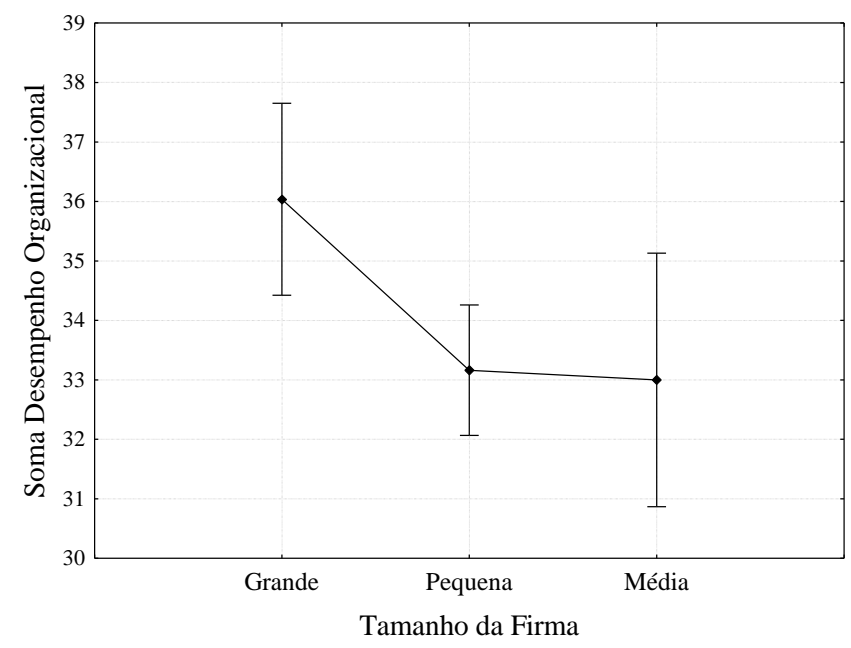

Figura 3 - Comparação simultânea na Anova da somatória da pontuação dos itens selecionados do desempenho organizacional segundo o tamanho do escritório de prestação de serviços contábeis

Fonte: Dados da pesquisa (2016)

E nas comparações pareadas com o teste de Tukey para número desigual se confirma que a diferença é significante aos $5 \%$, como se mostra na Tabela 3 , entre as médias da pontuação do desempenho para os escritórios grandes contrastados com os pequenos. Os de tamanho médio não se diferenciam dos grandes nem dos pequenos.

Tabela 3

Valores das pontuações médias e da significância das comparações do desempenho com o teste de Tukey para número desigual de elementos

\begin{tabular}{l|l|l|l}
\hline Tamanho & Grande & Pequena & Média \\
\hline Médias & 36,037 & 33,162 & 33,000 \\
\hline Grande & & 0,034837 & 0,115324 \\
\hline Pequena & 0,034837 & & 0,993798 \\
\hline Média & 0,115324 & 0,993798 & \\
\hline
\end{tabular}

Nota. Fonte: Dados da pesquisa (2016).

Com a finalidade de avaliar a primeira hipótese proposta organizou-se o modelo estrutural considerando as 5 dimensões do capital humano $(\mathrm{CH})$ com seus 23 itens influenciando o desempenho organizacional (DO), mensurado por 6 indicadores. Na Figura 4 exibe-se o modelo com os valores de covariância padronizada (ou correlações) das relações.

O ajustamento das relações por meio dos coeficientes de covariância foi obtido em 11 interações. Os resultados alcançados se apresentam na Tabela 4, em que se expõem os coeficientes padronizados e sua significância. 


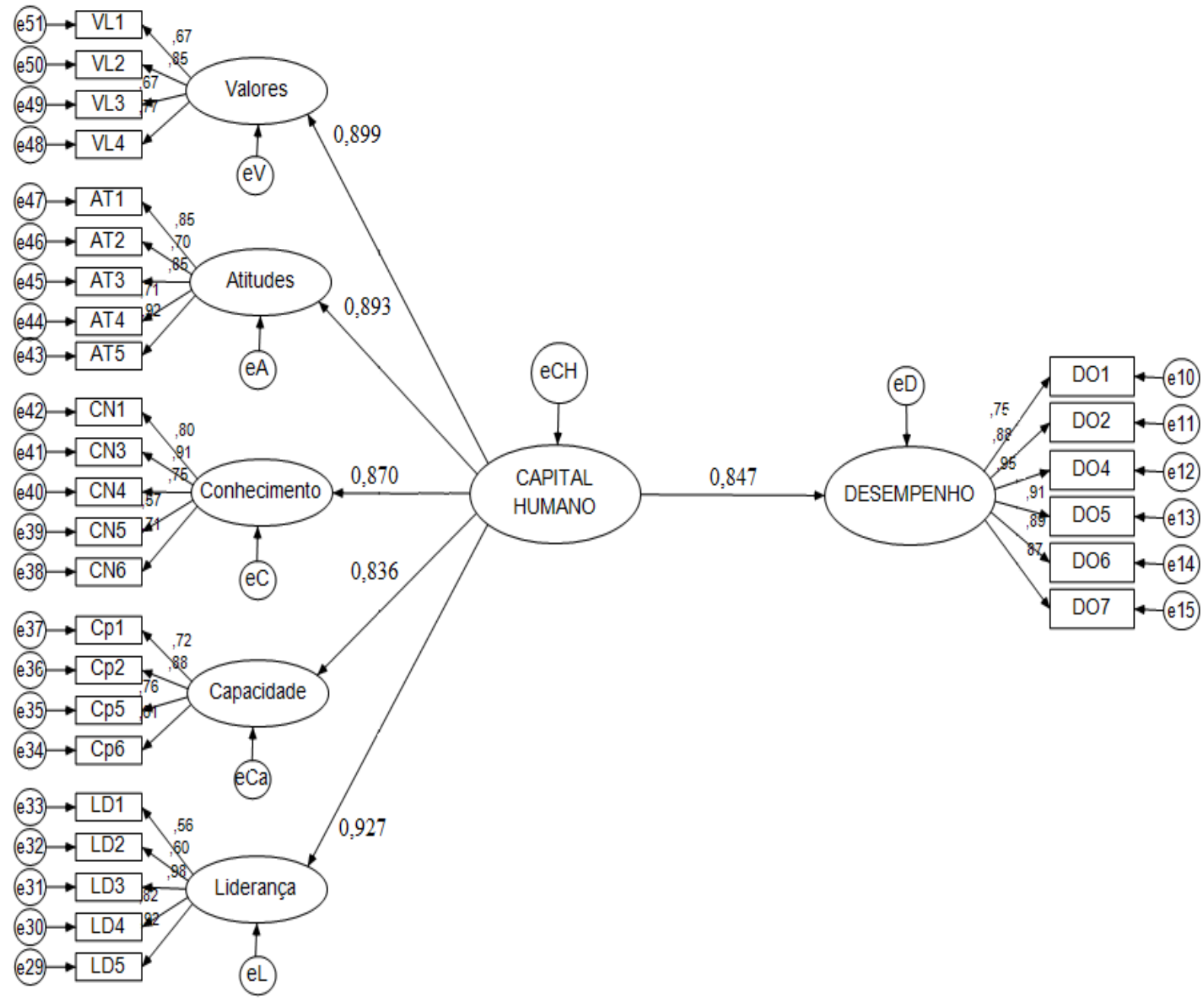

Figura 4. Modelo estrutural da relação entre o capital humano e o desempenho organizacional Fonte: Dados da pesquisa (2016).

Observando os valores tabelados confirma-se que todas as associações que têm o capital humano com suas dimensões e com o desempenho foram significativas.

Tabela 4

Coeficientes calculados na modelagem de equações estruturais e sua significância

\begin{tabular}{|c|c|c|c|}
\hline Relação & & Coeficiente & Significância \\
\hline Conhecimento & CAPITAL HUMANO & 0,87 & *** \\
\hline Atitudes & CAPITAL HUMANO & 0,893 & 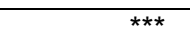 \\
\hline Capacidades & CAPITAL HUMANO & 0,836 & *** \\
\hline Valores & CAPITAL HUMANO & 0,899 & $\star \star * *$ \\
\hline Liderança & CAPITAL HUMANO & 0,927 & $* * *$ \\
\hline DESEMPENHO & CAPITAL HUMANO & 0,847 & 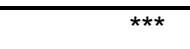 \\
\hline
\end{tabular}

Nota. Fonte: Dados da pesquisa (2016).

${ }^{* * *}: p<0.001$.

Confirma-se assim a hipótese que conjecturava o relacionamento positivo entre o $\mathrm{CH}$ e o DO. Esses mesmos achados foram obtidos nas pesquisas com diversos tipos de empresas, como nas do setor de software (Seleim et al. 2007) e o farmacêutico (Sharabati, 2013).

Para avaliar o ajustamento do modelo empregaram-se os seguintes índices: qui quadrado dividido pelos graus de liberdade ( $\mathrm{x}^{2} / \mathrm{g} . \mathrm{I}$.); Root Mean Square Error of Aproximation 
(RMSEA); Non-Normed Fit Index (NNFI); Comparative Fit Index (CFI); e, Root Mean Residual (SRMR). Os valores de referência sugeridos como adequados são: $X^{2} / g . l$ < < 5; RMSEA < 0,08; $\mathrm{NNFI}>0,90 ; \mathrm{CFI}>0,90 ; \mathrm{RMR}<0,10$.

No processamento dos dados dessa relação os valores de ajuste obtidos foram: $\chi^{2} / \mathrm{g} . \mathrm{l}$. = 5,5251; RMSEA $=0,086$; $\mathrm{NNFI}=0,852 ; \mathrm{CFI}=0,890$; e $\mathrm{RMR}=0,126$. Assim sendo o ajuste pode ser considerado satisfatório.

\section{CONCLUSÕES}

Em correspondência com o objetivo geral da pesquisa a investigação centrou-se na análise das associações entre o capital humano $(\mathrm{CH})$ e o desempenho organizacional (DO) dos escritórios de contabilidade. Com base no referencial teórico foi proposta a hipótese que postulava tal relação como positiva. Inicialmente fizeram-se algumas análises para saber se o gênero ou o tipo de gestão da empresa influenciava na percepção dos respondentes sobre os constructos.

Os testes de comparações de médias efetuados não mostraram diferenças significativas para nenhum desses preditores. Contudo, conforme o tamanho que as firmas têm constatou-se diferenças para o desempenho. As maiores são as que declaram ter melhor desempenho e ainda apresentaram média maior na pontuação do capital humano, com significância ao $10 \%$, se contrastadas com as empresas pequenas.

Como remarque conclusivo deste estudo ressalta-se que nos escritórios que fizeram parte da amostra o capital humano que dispõem relaciona-se positivamente com 0 desempenho que declaram ter comparativamente aos seus concorrentes. Ainda constatou-se que a dimensão que mais influencia nas relações com o capital humano é a liderança.

Sugere-se que outras pesquisas sejam desenvolvidas para esse tipo de organização considerando os outros componentes do capital intelectual, quais sejam o estrutural e o relacional. Também se recomenda que nas novas pesquisas seja ampliada a base de dados, incluindo outros constructos importantes, como a capacidade absortiva de conhecimentos, e efetuando uma amostragem probabilística, para assim poder generalizar os resultados.

\section{REFERÊNCIAS}

Backes, N. A., Silva, W. V. da, Adão, W. J., \& Corso, J. M. D. (2009). Indicadores contábeis mais apropriados para mensurar o desempenho financeiro das empresas listadas na BOVESPA. Revista Eletrônica de Estratégia \& Negócios, 2(1), 117-135.

Bontis, N. (2001). Assessing knowledge assets: a review of the models used to measure intellectual capital. International Journal of Management Review, 3(1), 41-60.

Bontis, N., Dragonetti, N., Jacobsen, K., \& Roos, G. (1999). The knowledge toolbox: a review of the tools available to measure and manage intangible resources. European Management Journal, 17(4), 391-402.

Bontis, N., Keow, W. C. C., \& Richardson, S. (2000). Intellectual capital and business performance in Malaysian industries. Journal of Intellectual Capital, 1(1), 85-100.

Bortoluzzi, S. C., Ensslin, S. R., \& Ensslin, L. (2010). Avaliação de desempenho dos aspectos tangíveis e intangíveis da área de mercado: estudo de caso em uma média empresa industrial. Revista Brasileira de Gestão de Negócios, 12(37), 425-446.

Brooking, A. (1996). Intellectual capital: core asset for the third millennium enterprise. Boston: Thomson Publishing Inc.

Cassol, A., Zapalai, J., \& Cintra, R. F. (2017). Capacidade absortiva como propulsora da inovação em empresas incubadas em Santa Catarina. Revista Ciência da Administração, 23(1), 9-41.

Chakravarthy, B. S. (1996). Measuring strategic performance. Management Journal, 7(5), 437447. 
Churchill, G. A., Jr. (1979). A paradigm for developing better measures of marketing constructs. Journal of Marketing Research, 16(1), 64-74.

Cohen, W. M., \& Levinthal, D. A. (1990). Absorptive capacity: a new perspective on learning and innovation. Administrative Science Quarterly, 35(1), 128-152.

Coleman, J. S. (1988). Social capital in the creation of human capital. The American Journal of Sociology, 94, Supplement, 95-120.

Curado, C., \& Bontis, N. (2007). Managing intellectual capital: the MIC matrix. International Journal of Knowledge and Learning, 3(2), 316-328, 2007.

Dani, A. C., Santos, C. A., Kaveski, I. D. S., \& Cunha, P. R. (2017). Características do conselho de administração e o desempenho empresarial das empresas listadas no Novo Mercado. Revista de Gestão, Finanças e Contabilidade, 7(1), 29-47.

De Castro, G. M., \& Sáez, P. L. (2008). Intellectual capital in high-tech firms: the case of Spain. Journal of Intellectual Capital, 9(1), 25-36.

Edvinsson, L., \& Malone, M. S. (1997). Intellectual capital at Skandia. Long Range Planning, 30(3), 266-373.

Edvinsson, L., \& Malone, M. S. (1998). Capital intelectual. São Paulo: Makron Books.

Finney, S. J., \& Distefano, C. (2006). Non-normal and categorical data in structural equation modeling. In: Hancok, G. R., \& Mueleer, R. O. Structural equation modeling: a second course. Charlotte, NC: Information Age Publ.

Florin, J. (2005). Is venture capital worth it? Effects on firm performance and founder returns. Journal of Business Venturing, 20(1), 113-136.

Gil, A. L., \& Arnosti, C. M. (2007). Balanço intelectual: a estratégia com projetos de mudanças e o reconhecimento dos talentos humanos. São Paulo: Saraiva.

Gracioli, C., Godoy, L. P., Lorenzett, D. B., \& Godoy, T. P. (2012). Capital intelectual: uma ferramenta inovadora na busca por vantagens competitivas. Revista de Administração e Inovação, 9(4), 96-120.

Gunasekaran, A., \& Kobu, B. (2007). Performance measures and metrics in logistics and supply chain management: a review of recent literature (1995-2004) for research and applications. International Journal of Production Research, 45(2), 2819-2840.

Hair, J. F., Jr., Black, W. C., Babin, B. J., Anderson, R. E., \& Tatham R. L. (2009). Análise multivariada de dados. (6a ed.) Porto Alegre: Bookman.

Harris, R. J. (1975). A primer of multivariate statistics. New York: Academic Press.

Hoque, Z. (2005). Linking environmental uncertainty to non-financial performance measures and performance: a research note. The British Accounting Review, 37, 471-481.

Huang, C. J., \& Liu, C. J. (2005). Exploration for the relationship between innovation, IT and performance. Journal of Intellectual Capital, 6(2), 237-252.

Igarashi, D. C. C., Ensslin, S. R., Ensslin, L., \& Paladini, E. P. (2008). A qualidade do ensino sob o viés da avaliação de um programa de pós-graduação em contabilidade: proposta de estruturação de um modelo híbrido. RAUSP, 43(2), 117-137.

Kamukama, N., Ahiauzu, A., \& Ntayi, J. M. (2010). Intellectual capital and financial performance in Uganda's microfinance institutions. African Journal of Acconting, Economics, Finance and Banking Research, 6(6), 17-31.

Kline, R. B. (2011). Principles and practice of structural equation modeling. (3a ed), New York/London: The Guilford Press. 
Lopes Costa, J. A. (2012). Capital intelectual e desempenho organizacional no sector hoteleiro português (nut ii Lisboa). Tese (doutorado europeu), Universidad de Extremadura, Espanha.

Martins, G. A., \& Theóphilo, C. R. (2007). Metodologia da investigação científica para ciências sociais aplicadas. São Paulo: Atlas.

Mcadam, R., \& Baile, B. (2002). Business performance measures and alignment impacto in strategy: the role of business improvement models. International Journal of Operations \& Production Management, 22(9), 972-996.

Neely, A., Gregory, M., \& Platts, K. (2005). Performance measurement system design: a literature review and research agenda. International Journal of Operations \& Production Management, 25(12), 1228-1263.

Oliveira, J. M., \& Beuren, I. M. (2003). O tratamento contábil do capital intelectual em empresas com valor de mercado superior ao valor contábil. Revista Contabilidade \& Finanças, 32, 81-98.

Pelham, A. M., \& Wilson, D. T. (1996). A longitudinal study of the impact of market structure, firm structure, strategy, and market orientation culture on dimensions of small- firm performance. Journal of Academy of Marketing Science, 24(1), 27-43.

Perez, M. M., \& Famá; R. (2006). Ativos intangíveis e o desempenho empresarial. Revista Contabilidade e Finanças, 17(40), 7-24.

Perin, M. G., \& Sampaio, C. H. (1999). Performance empresarial: uma comparação entre indicadores subjetivos e objetivos. Anais do Encontro Nacional dos Programas de Pós Graduação e Pesquisa em Administração, Foz do Iguaçu, PR, Brasil, 23.

Rahman, S. (2012). The role of intellectual capital in determining differences between stock market and financial performance. International Research Journal of Finance and Economics, 89(1), 46-77.

Sainaghi, R., \& Baggio, R. (2013). Structural social capital and hotel performance: is there a link? International Journal of Hospitality Management, accept 12 november 213 (last version before print).

Seleim, A., Ashour, A., \& Bontis, N. (2007). Human capital and organizational performance: a study of Egyptian software companies. Management Decision, 45(4), 789-801.

Sena, T. S., \& Petri, S. M. (2011). A relevância do cliente para um escritório de contabilidade: uma abordagem à compreensão do capital intelectual. Revista Catarinense da Ciência Contábil, 10(28), p. 41-57.

Sharabati, A. A. (2013). A the relationship between human capital and Jordanian Pharmaceutical organizations' business performance. International Journal of Academic Research in Business and Social Sciences, 3(1), 260-279.

Silva, R. Da, Bilich F., \& Gomes, L. F. A. M. (2002). Avaliação, mensuração e otimização de ativos intangíveis: utilização de método de apoio multicritério no capital intelectual. In: Anais do Encontro Nacional dos Programas de Pós Graduação e Pesquisa em Administração, Rio de Janeiro, RJ, Brasil, 36.

Stewart, T. (1998). Capital intelectual: a nova riqueza das organizações. Rio de Janeiro: Campus.

Sveiby, K. E. (1998). A nova riqueza das organizações: gerenciando e avaliando patrimônios de conhecimento. Rio de Janeiro: Campus.

Unger, J. M., Rauch, A., Frese, M., \& Rosenbusch, N. (2011). Human capital and entrepreneurial success: a meta-analytical review. Journal of Business Venturing, 26(3), 341-358.

Vergara, S. C. (1998). Projetos e relatórios de pesquisa em administração. São Paulo: Atlas. 
Wernke, R., Lembeck, M., \& Bornia, A. C. (2003). As considerações e comentários acerca do capital intelectual. Revista da FAE, 6(1), 15-26. 